\title{
Estudio de la enseñanza de ecosistemas amazónicos a través de la metodología de aprendizaje basado en la resolución de problemas (ABRP)
}

Study of the teaching of Amazonian ecosystems through the methodology of Problem-based learning (PBL)

\section{Carlos Henrique Nascimento ${ }^{1}$ Ires Paula de Andrade Miranda ${ }^{2}$}

\author{
${ }^{1}$ Estudiante del Doctorado en Biodiversidad y Conservación, Programa de Pos-Graduación en la Red Bionorte, Departamento de \\ Escuela Primaria, Semed, Manaos, AM, Brasil. \\ chsurvivor@hotmail.com
}

${ }^{2}$ Docente del Programa de Pos-Graduación de la Red Bionorte, Doctor en Ciencias Biológicas, Investigadora del Instituto Nacional de Pesquisa de la Amazonia (INPA), Manaos, AM, Brasil

iresmiranda@yahoo.com.br

\begin{abstract}
Resumen
La finalidad fue analizar el Aprendizaje Basado en la Resolución de Problemas (ABRP) como alternativa metodológica para la escuela primaria que favorezcan el aprendizaje sobre los ecosistemas amazónicos. Esta investigación es descriptiva con un enfoque cuali-cuantitativo. El estudio fue realizado con alumnos del 9o año de la escuela primaria. La metodología de enseñanza basada en el ABRP fue aplicada en dos fases: En la primera fase se realizó un test de concepciones previas para conocer la percepción de los alumnos sobre tópicos relacionados a algunas unidades de paisajes de los ecosistemas amazónicos. La segunda fase consistió en la ejecución de la metodología de aprendizaje en el ambiente escolar con alumnos del 9o año de la escuela primaria. Se establecieron cuatro fases distintas en la aplicación: i) selección de temas; ii) formulación de problemas; iii) resolución de problemas; iv) síntesis y evaluación. Los instrumentos de recolección de datos usados fueron: test de concepciones previas y cuadro de competencias. Además del diario del investigador y registros fotográficos. Los resultados demostraron que después de la aplicación de la metodología del $A B R P$, se puede percibir en los alumnos el reconocimiento cognitivo de los ecosistemas amazónicos, alcanzando metas adicionales que los PCN establecen.
\end{abstract}

Palabras-clave: Competencias cognitivas; Biodiversidad de la amazonia

\begin{abstract}
The purpose was to analyze the Problem-based learning (PBL) as a methodological alternative for primary school that favor learning about Amazonian ecosystems. This research is descriptive with a qualitative-quantitative approach. The study was carried out with students from the 9th year of primary school. The teaching methodology based on the PBL was applied in two phases: In the first phase, a test of previous conceptions was carried out in order to know the perception of the students on topics related to some units of landscapes of the Amazonian ecosystems. The second phase consisted of the implementation of the learning methodology in the school environment. Four different phases were established in the application: i) selection of topics; ii) problem formulation; iii) problem solving; iv) synthesis and evaluation. The data collection instruments used were: preconceptions test and skills chart. The results showed that after the application of the ABRP methodology, the cognitive recognition of the Amazonian ecosystems can be perceived in the students, reaching additional goals that the PCN establish.
\end{abstract}

Keywords: Cognitive skills; Amazonian biodiversity 


\section{Introducción}

La educación en ciencias de la enseñanza fundamental de acuerdo con los Parámetros Curriculares Nacionales ( $\mathrm{PCN}$ ) no enfatiza la necesidad cognitiva en la educación básica de estudios relacionados con las cuestiones ambientales dirigidas a la Amazonia y sus problemas ambientales (" información verbal).

En virtud de ello, el desarrollo de competencias para el aprendizaje basado en la resolución de problemas permite un abordaje cognitiva como facilitador de la interacción entre profesor y alumnos en un ambiente propicio relacionado al ecosistema donde esos actores están insertados.

A partir de ese indicio, esta investigación sugiere alternativas metodológicas para entender, como es que los alumnos que viven o nacen en la región amazónica perciben su contexto fitogeográfico como un ambiente distinto, dinámico y en continua actividad biológica.

Una alternativa encontrada fue el uso del aprendizaje basado en la resolución de problemas. El ABRP inicialmente se utilizó en la educación médica en Canadá, consiguiendo extenderse hasta Europa, principalmente en Portugal. Esta metodología enfatiza un enfoque dialéctico que busca crear situacionesproblemas generando un debate con el fin de encontrar soluciones hipotéticas (BARROWS, TAMBLYN, 1980).

El ABRP fue utilizado en diversas áreas como: medicina (BARROWS, TAMBLYN, 1980), educación superior (GÜRSES et al., 2007; BOUJAOUDE, 1992; HUFFMAN et al., 1997), química, bioquímica, matemática y física (CHANDRASEGARA et al., 2008; TARHAN et al., 2008; DODS, 1996; WHITE, 2001; GÜRSES et al., 2007; TAYLOR; MACDONALD, 2007) ciencias forenses, ciencias de la tierra (BELT et al., 2008; TARHAN et al., 2008; DODS, 1996; WHITE, 2001; GÜRSES et al., 2007, TAYLOR, MACDONALD, 2007) (2002), CHANG, 2002) como también en la formación de profesores (OSTERMAN Y KOTTKAMP, 1993).

Lo inédito de este trabajo no está en el área de concentración y sí en el contexto fitogeográfico, adicionalmente no fue encontrado en nuestra revisión de literatura investigaciones que traten asuntos de la relación del ABRP y los ecosistemas amazónicos. Lo que fundamenta la importancia de esta iniciativa como producción de conocimiento sobre el tema. La Amazonia tiene una posición estratégica en la política mundial de preservación ambiental. Conocer sus características contribuirá para que desde la enseñanza fundamental esa temática pueda traer a luz el interés en la preservación y conservación de su patrimonio genético.

La investigación fue desarrollada con 120 alumnos en la primera fase, donde fue posible identificar las concepciones previas de las clases sobre los ecosistemas amazónicos. En la segunda fase, se implementó la metodología del ABRP para 100 alumnos de la red pública de la escuela primaria municipal Jarlece da Conceição Zaranza en el municipio de Manaos, estado de Amazonas.

Se aplicó un cuestionario a los alumnos antes y después de la implementación del ABRP, de modo que los resultados demostraron en alguna medida que el uso del ABRP puede contribuir al conocimiento de los ecosistemas amazónicos.

En la fase de implementación se realizaron cinco periodos pedagógicos: I) formación de grupos y definición de temas; II) investigación en la red mundial de computadoras sobre los temas abordados; III) compartir y discutir sobre los temas investigados; IV) elaboración de situaciones-problemas y; V) presentación de las soluciones a las situaciones problemáticas.

Las dificultades encontradas en el desarrollo de la investigación fueron: acceso a la escuela de modo que no hubiera interferencia en el horario regular de las clases, la disponibilidad de los alumnos en permanecer y la disponibilidad de horarios alternativos para el curso de implementación del ABRP.

Otras dificultades que se dio durante el desarrollo de la investigación fue el dominio de la técnica en el uso del ABRP, desde su aplicación hasta recolección y análisis de los datos. La intención de dicho trabajo, no fue agotar el tema, como también evitar simplificar las conclusiones provenientes de las interpretaciones de los resultados en otros contextos que no hubiesen sido aplicados, siendo específicamente para los alumnos de la escuela primaria mencionada. 


\section{Características generales de las unidades de paisaje}

Hay varias clasificaciones sobre los ecosistemas amazónicos, pero, por una cuestión didáctica orientada a los alumnos de la escuela primaria trataremos los siguientes biomas: bosques húmedos, várzeas, campinaranas y sabanas. Modificando el mito del sentido común de que la Amazonia es homogénea.

En este sentido, cabe distinguir las distintas clasificaciones sobre la fitogeografía, la misma que sufrió varias clasificaciones a lo largo de la historia: clasificaciones históricas de Schimper (1903), Tansley y Chipp (1926), Burtt-Davy (1938), Dansereau (1968), Aubréville (1956), Trochain (1969), Ellenberg y Mueller -Dormales (1967) y Di Glegorio (2005); clasificación continental de Beard (1955) y Morrone (2001); clasificación brasileña de Martius (2012), Campos (1926), Sampaio (1943), Azevedo (1950), Rizzini (1979), Eiten (1983), Fernandes (1998) y Morrone (2001).

Los aspectos fisionómicos, climáticos y edáficos están presentes en las clasificaciones de Schimper (1903), Tansley (1926), Burtt-Davy (1938) y Mueller-Dombois (1967). Los aspectos ecológicos se incorporan en la clasificación de Dansereau (1968). Los aspectos altimétricos se destacan en la clasificación de Aubréville (1956) y florísticos en la clasificación de Trochain (1969).

La clasificación de Di Glegorio (2005) tiene en cuenta un conjunto de atributos de la cobertura, llamados "clasificadores" que están dispuestos jerárquicamente según el nivel de clasificación. Las clasificaciones brasileñas más modernas como el proyecto RADAM (2010) son las primeras iniciativas de comprender la fitogeografía de la región amazónica.

\subsection{Bosques tropicales húmedos}

En el caso de los bosques tropicales, el bosque húmedo se caracteriza por períodos largos de lluvia, constituidos por vegetación de alto porte con estratos bien definidos (herbáceos, arbustivos y arbóreos). Sin embargo, la altura de los árboles impide que la luz del sol penetre en el interior del bosque, limitando así el índice de regeneración natural del dosel inferior.

Los bosques tropicales húmedos o pluviales están constituidos por especies latifoliadas y perennes que ocupan zonas de baja latitud, próximas al ecuador, donde la duración de los días se mantiene casi constante a lo largo del año (RIZZINI, 1979).

Por su parte la precipitación anual supera los $2.000 \mathrm{~mm}$, pero no siempre está bien distribuida, pudiendo haber algunos períodos relativamente secos. Estos bosques húmedos ocurren en América del Sur, la cuenca amazónica y el río Orinoco, y también en Centroamérica, en el centro-oeste de África y Madagascar y en ciertas regiones de la India, Malasia, Borneo y Nueva Guinea (IBGE, 1993).

\subsection{Bosque húmedo}

Los bosques de várzeas están presentes en las planicies de inundación. Donde los sedimentos, partículas en suspensión de material arcilloso (agua blanca y greda), están siendo depositados a partir del Holoceno (hay 10 mil a.a.). El flujo de las mareas eleva el nivel del agua entre 2 a 4 metros. Los bosques de várzea se subdividen en várzeas de mareas y várzeas estacionales (IBGE, 1993).

Las várzeas son estacionales cuando sufren influencia directa del ciclo anual de inundación. Según PRANCE (1979), la riqueza de especies en los bosques de várzea es menor que en la tierra firme. La composición florística de las várzeas se constituye en plantas acuáticas, plantas leñosas, arbustos y lianas. El paisaje de las áreas inundables es resultado de la dinámica de los sistemas hidro-geomorfológicos de los ríos (MEADE, 1994).

\subsection{Sabanas}

Algunos autores usan las denominaciones de "estirpe", "cerrado" o "campo del cerrado" como sinónimos de sabana (COLE, 1963, 1986). Según el IBGE (1993) algunos autores clasifican las sabanas en cuatro subgrupos: a) sabana forestal; b) sabana arbolada (campo cerrado, cerrado ralo, cerrado típico y 
cerrado denso); c) sabana parque (campo sucio del cerrado, cerrado del pantanal, campo rupestre); y d) sabana gramínea leñosa (campo limpio del cerrado).

La sabana forestal son áreas arenosas con suelos lixiviados y profundos. Presencia de leñosas micro y nano-fanerófitos de altura variable entre 6 a $8 \mathrm{~m}$. Su composición florística posee géneros Caryocar, Salvertia, Boudichia, Dimorphandra, Qualea, Anadenanthera, Kielmeye.

La sabana arbolada (campo cerrado, cerrado ralo, cerrado típico y cerrado denso) presenta fisonomía nano-fanerofítica rala y otra hemicriptofítica de gramínea continúa. Su composición florística contiene géneros Salvertia, Curatella, Himatanthus, Parkia, Platonia, Stryphnodendron.

La sabana parque (campo sucio del cerrado, cerrado del pantanal, campo rupestre) posee estratos graminoides, integrados por hemicriptófitos y geófitos de florística natural antrópica entremezclada por nano-fanerófitos aislados similares al "parque Inglés". Ocurre en campos latosos y/o rupestres. En áreas empapadas de depresiones inundadas. Su composición florística posee los géneros Hancornia, Handroanthus, Byrsonima.

La sabana gramínea leñosa (campo limpio del cerrado) ocurre en áreas dominadas por hemicriptófitos y su composición florística tiene los géneros Andira, Chamaecrista, Byrsonima, Attalea, Orbignya, Axonodus, Andrógino, Aristida, Echinolaena, Paspalum, Trachypogon, Schizachyrium, Tristachya.

\subsection{Campinaranas}

El IBGE (1993) propone Caatinga de la Amazonia, Caatinga gapó o Campina de la Amazonia como sinónimos de Campinaranas. Según Kuhlmann (1977) es una región ecológica, campestre con su clímax edáfico de área boscosa y etapas sucesoras arbóreas, arbustivas y gramíneas leñosa.

Las campinaranas ocupan áreas lixiviadas y húmedas con suelos cuaternarios de clima cálido con precipitaciones superiores a $3000 \mathrm{~mm}$ anuales y medias de temperaturas de $25^{\circ} \mathrm{C}$.

Las campinaranas se dividen en cuatro subgrupos: a) arbórea densa (Caatinga de la Amazonia y caatinga-gapó); b) campinaranas arboladas; c) campinaranas arbustivas; y d) campinaranas gramíneasleñosa.

La campinarana arbórea densa (Caatinga de la Amazonia y caatinga-gapó) se caracteriza por áreas planas tabulares de acumulaciones arenosas inundables. En el caso de la vegetación nueva o "raparía", reciben el nombre de "resaca" y su composición florística posee géneros Clusia, Aldina, Hevea, Enriquezia, Eperia, Caraipa.

Ya la porción de la Caatinga-gapó compuesta de áreas de bosque inundado, deprimidas y llanuras de ríos de agua negra. Suelos poco extensos con árboles finos, troncos rectilíneos, cáscaras sueltas y claras con altura de hasta $20 \mathrm{~m}$. Composición florística contiene los géneros: Mauritia, Mautitiella, Leptocaryum y las familias Bromoliáceas y orquidáceas.

Las campinaranas arboladas están constituidas de árboles en áreas de interfluvios tabulares y llanuras fluviales con acumulaciones arenosas. Terrenos toreados por esporos. Su composición florística contiene géneros Trichomonas, Humeria, Astrocarium, Leopoldina, Euterpe, Cladonia.

Las campinaranas arbustivas tienen la presencia de arbustos, hierbas y árboles bajos que ocurren en áreas de depresiones cerradas con composición florística semejante a las campinaranas arboladas, sin embargo la altura de los árboles raramente sobrepasa los $2 \mathrm{~m}$.

Las campinaranas gramíneas leñosa tienen formación herbácea. Ocurren en llanuras empapadas de ríos de agua negra. Presentan áreas de periodos lluviosos, con amplia ocurrencia de incendios forestales. Su composición florística contiene géneros Palpalanthus, Drossera y Familias Poaceae, Cyperaceae, Amarylidaceae, Xyridaceae y Orchidaceae.

\section{El aprendizaje basado en la resolución de problemas (ABRP)}

El modelo de aprendizaje basado en la resolución de problemas (ABRP) o Problem-based Learning (PBL) fue delineado por sus pioneros en la Universidad de Macmaster, en Canadá. Parte de la premisa de que es posible llegar al conocimiento integrado a través de enfoques en que los alumnos son estimulados a reflexionar sobre problemas, y a buscar la información de que carecen para poder llegar a 
sus posibles soluciones, con ello ampliando su base de conocimiento conceptual y procesal (BARROWS, TAMBLYN, 1980).

Introducida en los años 1980 en los currículos de medicina de América del Norte. En la actualidad, su aplicación ha sido extendida a todo el mundo y con abordaje en varias disciplinas. El éxito en la Educación en Medicina se escudriñó el camino para su implementación en la Educación en Ciencias, principalmente en la enseñanza superior (GÜRSES et al., 2007; BOUJAOUDE, 1992; HUFFMAN et al., 1997), pero también en la enseñanza básica y secundaria (VASCONCELOS, 2012).

La investigación revela el éxito de la ABRP en áreas como la química (CHANDRASEGARA et al., 2008, TARHAN et al., 2008), la bioquímica (DODS, 1996, WHITE, 2001), las Ciencias Forenses (BELT et al., 2002, la Física (GÜRSES et al., 2007), las Ciencias de la Tierra (CHANG, 2002), la Matemática (TAYLOR, MACDONALD, 2007) y la Educación Ambiental (VASCONCELOS, 2008; 2012).

La teoría que preconiza la ABRP puede ser rastreada hasta la teoría de John Dewey (1938) del constructivismo y su creencia en la unidad de teoría y práctica. Dewey también creía que el aprendizaje debería ser activo y que los niños debían ir a la escuela para cumplir las obligaciones estudiantiles. Él sugirió que la educación debía involucrarse con la experiencia para expandirla. Los métodos utilizados para educar deberían proporcionar competencias de explotación, pensamiento y reflexión.

Siendo la interacción con el ambiente necesario para el aprendizaje. La democracia debe ser acogida en el proceso educativo. Dewey defendió el proceso de aprendizaje experiencial a través de la vida real para construir el conocimiento condicional, lo que es consistente con la teoría del constructivismo. Él propuso que el aprendizaje debía ser anclado en la educación por experiencias (DEWEY, 1938).

John Dewey (1859-1952), fue un filósofo de la educación estadounidense impregnado de influencias de Hegel y William James. Él se titulaba pragmatista experimental de la línea filosófica que se conoció como instrumentalismo. Preconizaba la fundación de la cognición activa o en experiencias no cognitivas tradicionales. Según William James, el pragmatismo es:

El pragmatismo defiende que el sentido de todo está en la utilidad - o efecto práctico - que cualquier acto, objeto o proposición pueda ser capaz de generar. Una persona pragmatista vive por la lógica de que las ideas y actos de cualquier persona solamente son verdaderos si sirven a la solución inmediata de sus problemas (COLL; SOLE, 1988).

El método de investigación de Dewey o metodología instrumentalista como proceso de investigación pasa de una situación indeterminada que bloquea una acción para una situación determinada que la acción puede seguir adelante.

Sus niveles de investigación son: i) definir el problema a través de la observación y el análisis; ii) construcción imaginativa de hipótesis para explicar y resolver el problema; iii) explicación del sentido de los conceptos en las hipótesis visando formulaciones matemáticas, desarrollo experimental y posteriores deducciones; iv) prueba real (BROWN et al, 2002).

Se nota a continuación cómo las características del ABRP son influenciadas por el método de investigación instrumentalista de la epistemología de Dewey. La literatura no deja claro las etapas secuenciales del ABRP, pero podemos describir sus características. El ABRP posee momentos de i) investigación u observación de la realidad: los alumnos son llevados a observar la realidad con el objetivo de captar los diferentes aspectos que la envuelven; ii) análisis o teoría del problema: los alumnos analizan la parte teórica del problema buscando saber el ¿por qué de las cosas observadas?; iii) evaluaciones de las soluciones o formulación de hipótesis para la solución del problema: el alumno es llevado a pensar con el fin de proporcionar respuestas hipotéticas al problema observado cultivando la originalidad y la creatividad (BERBEL, 1999).

Esas etapas o momentos se realizan en el ambiente que llamamos escenario problemático, que específicamente es la presentación clara y concisa del problema a ser estudiado, pudiendo ser utilizados recortes de noticias, vídeos, fotos o maquetas de contextos reales para análisis.

La selección de ese escenario es el momento más importante de la metodología, pues, a partir de ahí posibilitará o no toda la argumentación e hipótesis para la solución del problema 
(LAMBROS, 2002). Un escenario problemático simple, posiblemente generará cuestionamientos más directos que resultaran en respuestas del tipo "sí" o "no" (LECHE, PALMAS, 2006, CARVALHO, DOURADO, 2009).

Según Dahlgren y Oberg (2001) las cuestiones que respaldan un desarrollo cognitivo elevado son las siguientes: i) cuestiones de comprensión; ii) cuestiones de relación; iii) cuestiones de evaluación; iv) cuestiones de búsqueda por solución.

\section{4 material y métodos}

El estudio fue realizado con alumnos del $9^{\circ}$ año de la escuela primaria municipal Jarlece da Conceição Zaranza, de la Ciudad de Manaos, Amazonas.

La metodología de enseñanza basada en el ABRP fue aplicada en dos fases: En la primera fase se realizó una prueba de concepciones previas para conocer la percepción de los alumnos sobre tópicos relacionados a algunas unidades de paisajes de los ecosistemas amazónicos: i) bosque tropical; ii) várzea; iii) campinaranas y; iv) sabanas.

La segunda fase o fase de implementación consistió en la ejecución de la metodología de aprendizaje en el ambiente escolar con alumnos del 9o $^{0}$ ano de la educación primaria. Se establecieron cuatro fases distintas en la aplicación: i) selección de temas; ii) formulación de problemas; iii) la resolución de los problemas; iv) síntesis y evaluación.

Los instrumentos de recolección de datos usados fueron: prueba de concepciones previas, informe socioeconómico y cultural de los alumnos (RSECA), cuadro de competencias (cognitivas, comunicación, procesales y actitudinales). Además del diario del investigador y registros fotográficos.

\section{Resultados}

\subsection{Fase de concepciones previas}

Tabla 1 - Respuestas de la cuestión 1 de las concepciones previas de los alumnos de la Escuela Municipal Jarlece da Conceição Zaranza, Manaos, AM

\begin{tabular}{|c|c|}
\hline ALUMNOS & CONCEPCIONES PREVIAS \\
\hline A1 & "Sistema para ayudar a la naturaleza o hablar del bosque" \\
\hline A5 & "Sistema solar" \\
\hline A7 & "No, nada, me olvidé" \\
\hline A8 & "La mayor selva tropical del mundo" \\
\hline A10 & "Dejó en blanco" \\
\hline A11 & "Ecología y el sistema" \\
\hline A18 & "Ecosistema importante" \\
\hline A19 & "Bosque donde hay varias especies de animales" \\
\hline A20 & "Como es hermoso el lugar y los bichos" \\
\hline A25 & "Varias selvas y plantas" \\
\hline A28 & "Bosque" \\
\hline A32 & "Bosques" \\
\hline A33 & "Bosques, montañas" \\
\hline A35 & $\begin{array}{l}\text { "Ecología, naturaleza, problemas ecológicos, prevención con la } \\
\text { naturaleza" }\end{array}$ \\
\hline A36 & "Bosques, cuidado con los bosques, lluvia y animales" \\
\hline A40 & "Naturaleza, fauna y flora" \\
\hline A55 & "Sol, aire, luz" \\
\hline A58 & "Conjunto de bosque" \\
\hline A60 & "Ecología" \\
\hline A80 & "Planeta en las líneas, sin contaminación, sin basura" \\
\hline
\end{tabular}


Se aplicaron los cuestionarios propuestos en la metodología con las siguientes cuestiones: En la pregunta 1, se preguntó a los alumnos de la escuela primaria: Cuándo hablamos sobre el ecosistema ¿Qué es lo que usted se imagina? Y ellos respondieron de una manera simple y genérica. A continuación, se destacan algunas de las respuestas:

En la pregunta 2, se preguntó a los alumnos de la Enseñanza Fundamental: ¿Los ciudadanos de Manaos, qué pensamos sobre la Amazonia? Se constató respuestas simples, revelando superficialidad sobre los conceptos relacionados con la Amazonia.

Y algunos usan grandes "clichés" que son reproducidos por los medios de comunicación impresos o televisivos. Se destacan algunas respuestas que ilustran el punto de vista de los medios de gran masa:

Tabla 2 - Respuestas de la cuestión 2 de las concepciones previas de los alumnos de la Escuela Municipal Jarlece da Conceição Zaranza, Manaos, AM

\begin{tabular}{|l|l|}
\hline \multicolumn{1}{|c|}{ ALU } & CONCEPCIONES PREVIAS \\
\hline MNOS & "Lleno de árboles" \\
\hline A1 & "Un bosque" \\
\hline A5 & "El mayor bosque tropical del mundo, más de un tercio de animales del mundo, un bosque \\
\hline A7 & salvaje que la evolución corre deliberadamente" \\
\hline A8 & "Nunca fui allí" \\
\hline A10 & "La mayor selva tropical del mundo, paisajes hermosos, árboles y especies de animales" \\
\hline A11 & "Vemos la amazonia como un tesoro" \\
\hline A18 & "Un lugar con muchas cosas buenas para hacer" \\
\hline A19 & "Gran bosque con varios tipos de árboles y animales" \\
\hline A20 & "Lugar muy bonito, muy grande y frío" \\
\hline A25 & "Árbol, bosques y plantas" \\
\hline A28 & "Monte y árboles" \\
\hline A32 & "Bosques, matorrales, árboles, pisos húmedos, bichos y perezosos" \\
\hline A33 & "Nuestro bien material más precioso" \\
\hline A35 & "El bosque más grande considerado como pulmón del mundo" \\
\hline A36 & "Lugar peligroso, muchos animales, varios tipos de árboles, un gran bosque inmenso" \\
\hline A40 & "Bosque, agua, animales" \\
\hline A55 & "Muchos árboles y matorrales" \\
\hline A58 & "Amazonia es un bosque" \\
\hline A60 & "Muy caliente y agua" \\
\hline A80 & "Muchos bosques" \\
\hline
\end{tabular}

En la pregunta 3, se preguntó a los alumnos de la escuela primaria: ¿Cuáles son los ambientes de la Amazonia que usted conoce? Las respuestas de los alumnos de la escuela primaria, siguen realidad y contextos homogéneos, se destacaron las siguientes en el tabla 3:

Tabla 3 - Respuestas de la cuestión 3 de las concepciones previas de los alumnos de la Escuela Municipal Jarlece da Conceição Zaranza, Manaos, AM

\begin{tabular}{|l|l|}
\hline ALUMNOS & CONCEPCIONES PREVIAS \\
\hline $\mathbf{A 1}$ & "Bosques, animales y etc ..." \\
\hline $\mathbf{A} 5$ & "Sí, pero no recuerdo ..." \\
\hline $\mathbf{A 7}$ & "Ambientes de árboles enormes" \\
\hline $\mathbf{A} 8$ & "Frío, aireado y refrescantes" \\
\hline $\mathbf{A 1 0}$ & "Monte Roraima, jardín botánico de Manaos" \\
\hline $\mathbf{A 1 1}$ & "Conozco, pero no sé los nombres ..." \\
\hline $\mathbf{A 1 8}$ & "Medio ambiente húmedo, caliente y bastante sofocado" \\
\hline $\mathbf{A 1 9}$ & "Río negro, encuentro de las aguas" \\
\hline
\end{tabular}


En la Tabla 4, muestra que el 65\% de los alumnos entrevistados conocen algún tipo de ambiente en la Amazonia. Mientras el 35\% de los alumnos entrevistados no conocen ambientes estos ambientes. Siendo $n$ poblacional igual a 120 alumnos entrevistados. A continuación en la tabla 4, qué ambientes conocen los alumnos:

Tabla 4 - Respuestas de la cuestión 1 de las concepciones previas de los alumnos de la Escuela Municipal Jarlece da Conceição Zaranza, Manaos, AM

\begin{tabular}{|c|c|}
\hline Respuesta & Porcentaje \\
\hline Conozco & $65 \%$ \\
\hline Desconozco & $35 \%$ \\
\hline
\end{tabular}

En la pregunta 4, ¿Has oído hablar de un bosque húmedo? Si es así, ¿Cuál es la apariencia de este tipo de bosque? En la tabla 5, los alumnos entrevistados revelan que el 50\% de los alumnos ya escucharon hablar sobre el bosque húmedo.

Tabla 5 - Respuestas de la cuestión 4 de las concepciones previas de los alumnos de la Escuela Municipal Jarlece da Conceição Zaranza, Manaos, AM

\begin{tabular}{|c|c|}
\hline Respuesta & Porcentaje \\
\hline Si & $50 \%$ \\
\hline No & $50 \%$ \\
\hline
\end{tabular}

En la tabla 6, sólo muestra las respuestas de aquellos alumnos que ya escucharon hablar del bosque húmedo destacando ¿cuál es su apariencia de este tipo de bosque?

Tabla 6 - Respuestas de la cuestión 4 de las concepciones previas de los alumnos de la Escuela Municipal Jarlece da Conceição Zaranza, Manaos, AM

\begin{tabular}{|l|l|}
\hline ALUMNOS & CONCEPCIONES PREVIAS \\
\hline $\mathbf{A 1}$ & "Ambiente limpio y agradable" \\
\hline $\mathbf{A 5}$ & $\begin{array}{l}\text { "Bosque que las aguas de la lluvia se } \\
\text { evaporan antes de caer al suelo, deja la } \\
\text { tierra húmeda, fría o caliente" }\end{array}$ \\
\hline $\mathbf{A 7}$ & "Pero no sé describir" \\
\hline $\mathbf{A 8}$ & "Hojas, árboles todos húmedos" \\
\hline $\mathbf{A 1 0}$ & $\begin{array}{l}\text { "Árboles altos grandes bastante verde con } \\
\text { muchos animales" }\end{array}$ \\
\hline $\mathbf{A 1 1}$ & "Bosque con mucha lluvia" \\
\hline $\mathbf{A 1 8}$ & "Medio húmedo y medio seco" \\
\hline
\end{tabular}

En la pregunta 5, ¿Has escuchado hablar de várzea? Si es así, ¿Cuál es su la apariencia de este tipo de ecosistema? En la tabla 6, muestra que todos los alumnos entrevistados en el total de 120, no conocen o nunca escucharon hablar de várzea. 
Tabla 6 - Respuestas de la cuestión 5 de las concepciones previas de los alumnos de la Escuela Municipal Jarlece da Conceição Zaranza, Manaos, AM

\begin{tabular}{|c|c|}
\hline Respuesta & Porcentaje \\
\hline Si & $0 \%$ \\
\hline No & $100 \%$ \\
\hline
\end{tabular}

En la pregunta 6, ¿has escuchado hablar de campinaranas? Si es así, ¿Cuál es la apariencia de este tipo de ecosistema? En la tabla 7, muestra que todos los alumnos entrevistados en el total de 120, no conocen o nunca escucharon hablar de campinaranas.

Tabla 7 - Respuestas de la pregunta 6 de las concepciones previas de los alumnos de la Escuela Municipal Jarlece da Conceição Zaranza, Manaos, AM

\begin{tabular}{|c|c|}
\hline Respuesta & Porcentaje \\
\hline Si & $0 \%$ \\
\hline No & $100 \%$ \\
\hline
\end{tabular}

En la pregunta 7, ¿has oído hablar de sabanas? Si es así, ¿Cuál es la apariencia de este ecosistema? En la tabla 8, muestra que todos los alumnos entrevistados en el total de 120, no conocen o nunca escucharon hablar de sabanas.

Tabla 8 - Respuestas de la pregunta 7 de las concepciones previas de los alumnos de la Escuela Municipal Jarlece da Conceição Zaranza, Manaos, AM

\begin{tabular}{|c|c|}
\hline Respuesta & Porcentaje \\
\hline Si & $0 \%$ \\
\hline No & $100 \%$ \\
\hline
\end{tabular}

\subsection{Resultados de la implementación de la metodología ABRP}

\subsubsection{Aula 01}

Figura 1 - Aula 01 - formación de grupos y sorteos de los temas de los alumnos de la fase de implementación de la metodología del ABRP

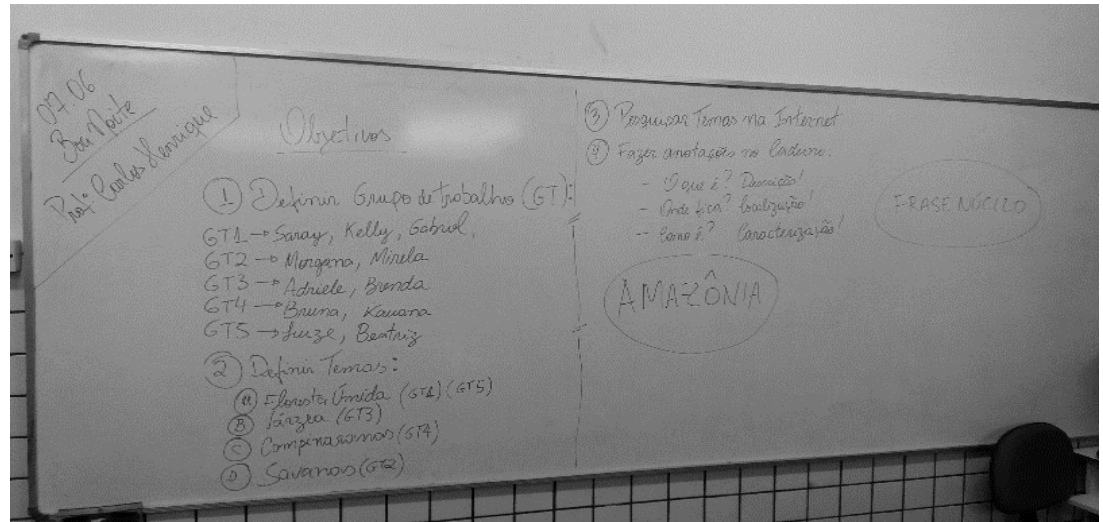

Fonte: Elaborada pelo autor 
En el segundo semestre de 2017. Se presentó el proyecto de tesis, e inmediatamente se definieron los grupos de trabajo con cuatro grupos de cinco cursos, siendo cada grupo compuesto por cinco alumnos, (tabla abajo) y a continuación el sorteo de los temas sobre los ecosistemas amazónicos. Los registros de datos se dieron por el diario del investigador (DI) y la aplicación del RSECA.

Tabla 9 - Esquema de codificación de los grupos y clases de los alumnos de la fase de implementación de la metodología del ABRP

\begin{tabular}{|c|c|c|c|c|}
\hline \multicolumn{2}{|c|}{ CLASES } & \multicolumn{4}{|c|}{ GRUPOS } \\
\hline T1 & T1G1 & T1G2 & T1G3 & T1G4 \\
\hline T2 & T2G1 & T2G2 & T2G3 & T2G4 \\
\hline T3 & T3G1 & T3G2 & T3G3 & T3G4 \\
\hline T4 & T4G1 & T4G2 & T4G3 & T4G4 \\
\hline T5 & T5G1 & T5G2 & T5G3 & T5G4 \\
\hline
\end{tabular}

\subsubsection{Diario del investigador (DI) - clase 01}

El investigador diario (DI) mostró los siguientes resultados: en el primero, tema I, II, VII no fue necesario responder de acuerdo con el tiempo de enseñanza. En la parte 1, punto III, se dio cuenta de que los grupos se crean de acuerdo con afinidades. El líder fue auto declarado y el mismo distribuía las tareas en el grupo. Quejas posteriores revelaron que los estudiantes no cooperan en el grupo como una cuestión de afirmación o falta de atención (T1G4, T2G3, T3G1, T3G2, T3G3, T4G1, T4G3, T4G4, T5G1, T5G1, T5G2, T5G3).

En la parte 1, punto IV, los problemas observados en los grupos eran la manera en la que se tomaron las decisiones, sin mayores complicaciones. En la pregunta V, las dudas se daban por la nomenclatura de los temas sorteados. Se notó una búsqueda por parte de los alumnos, para transmitir una respuesta apropiada (T5G4).

En la cuestión VI, no hubo opiniones extrañas porque se trataba de un momento inicial.

\subsubsection{Aplicación del RSECA - clase 01}

Tabla 10 - Las respuestas a las profesiones RSECA Campo de aplicación de los estudiantes de la fase de implementación de la metodología ABRP

\begin{tabular}{|c|c|}
\hline Respuesta & Porcentaje \\
\hline Categoría 1 & $37 \%$ \\
\hline Categoría 2 & $29 \%$ \\
\hline Categoría 3 & $13 \%$ \\
\hline Categoría 4 & $21 \%$ \\
\hline Categoría 5 & $0 \%$ \\
\hline Categoría 6 & $0 \%$ \\
\hline
\end{tabular}


Tabla 11 - Respuestas a la aplicación de la escala de habilidades académicas del RSECA de los alumnos de la fase de implementación de la metodología de la ABRP

\begin{tabular}{|c|c|}
\hline Respuesta & Porcentaje \\
\hline Categoría 1 & $9 \%$ \\
\hline Categoría 2 & $3 \%$ \\
\hline Categoría 3 & $5 \%$ \\
\hline Categoría 4 & $33 \%$ \\
\hline Categoría 5 & $45 \%$ \\
\hline Categoría 6 & $5 \%$ \\
\hline
\end{tabular}

\subsubsection{Aula 02}

Figura 2 - Aula 02: investigación de los temas por los alumnos en la fase de implementación de la metodología del ABRP

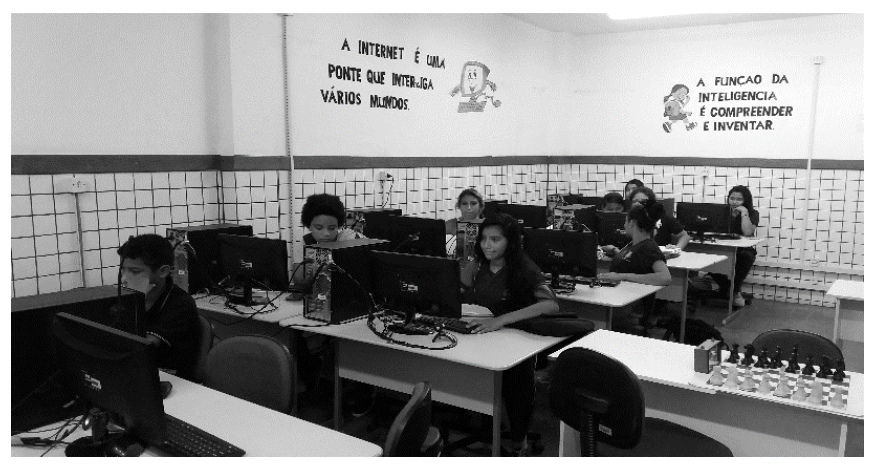

Fonte: Elaborado pelo autor

Siguiendo el plan del curso, la clase 02 fue realizada en el segundo semestre de 2017. Donde los alumnos realizaron la investigación de los temas en la red mundial de computadoras. El profesor supervisó la investigación y ayudó a filtrar las informaciones más pertinentes del tema, haciendo énfasis en ¿cómo es que los ecosistemas tienen el sesgo amazónico? El registro de datos se dio por el DI y por la observación de las competencias.

\subsubsection{Diario del investigador (DI) - clase 02}

El diario del investigador (DI) reveló los siguientes resultados: en la primera parte, cuestión I, no hubo necesidad de respuesta porque no se trataba del momento de debate de los temas. En el caso II, los grupos T1G3, T1G4, T2G4, T3G4, T4G1, T4G2, T4G4, T5G2 presentaron dificultades para investigar y seleccionar la información pertinente a los temas. Desde el distanciamiento del tema, dominio de las herramientas de investigación, funcionamiento de la red mundial de computadoras y la regionalización de los temas.

En las cuestiones III y IV no hubo cambios en la conducta de los líderes de los grupos en relación a la clase 01. En la pregunta V, las clases 4 y 5 por tratar de alumnos con mayor serie de distorsión presentaron muchos problemas en relación a la nomenclatura de los temas y la regionalización de los temas.

En la cuestión VI, las cuestiones más interesantes eran en relación a las diferencias climáticas y vegetación de las sabanas africanas en relación a las sabanas amazónicas. 
En la cuestión VII, no coincide la clase 02 porque no hubo presentación de los alumnos.

\subsubsection{Observación de las competencias - clase 02}

Tabla 12 - Observación de las competencias de los alumnos en la clase 02 de la fase de implementación de la metodología del ABRP

\begin{tabular}{|c|c|c|c|c|c|c|}
\hline Requisitos & (A) & (B) & (C) & (D) & (E) & (F) \\
\hline T1 & S & S & S & S & S & N \\
\hline T2 & S & S & S & S & S & N \\
\hline T3 & S & S & S & S & S & N \\
\hline T4 & S & S & S & N & N & N \\
\hline T5 & S & S & N & N & N & N \\
\hline
\end{tabular}

Hubo un cambio en la estrategia de recolección de datos en ese momento, en vez de recoger las informaciones de las observaciones de competencias de una manera individual, estamos hablando del universo $\mathrm{n}=100$, se modificó para observar las competencias por clases, a través del registro partiendo del observador participante. Como el instrumento de recolección de datos de observación de las competencias se restringe a las respuestas sí $(\mathrm{S})$ o no $(\mathrm{N})$, se consideró la respuesta $(\mathrm{N})$ para toda la clase la existencia de por lo menos un caso en los grupos.

\subsubsection{Aula 03}

Figura 3. Aula 03 - Discusión y elaboración de las situaciones-problemas por los alumnos en la fase de implementación de la metodología del ABRP

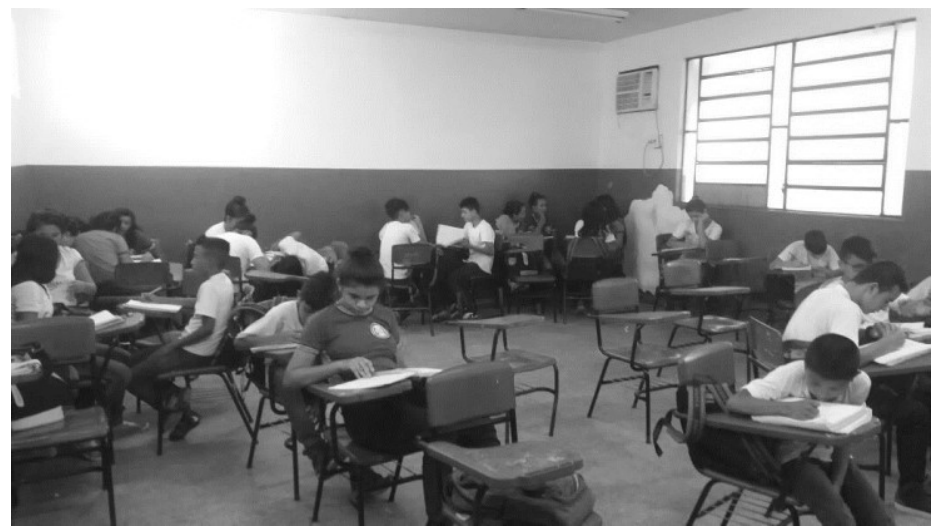

Fonte: Elaborado pelo autor

En el segundo semestre del 2017. Donde los alumnos realizaron compartimentos y estudio de la investigación de los temas en la red mundial de computadoras ocurrida en la clase 02. El profesor supervisó en el sentido de optimización del tiempo y el periodo de la clase, adicionalmente, orientó a cómo hacer la lectura e interpretación de los textos investigados y cuestionó a los alumnos sobre las contradicciones de la investigación. El registro de datos se dio por el DI y la observación de las competencias.

\subsubsection{Diario del investigador (DI) - clase 03}

El diario del investigador (DI) reveló los siguientes resultados: en la primera parte, cuestión I, de una manera general, todas las clases presentaron bastante dificultad en comprender los conceptos 
involucrados en los temas investigados, porque para la mayoría se trata del primer contacto. Realmente, aquí podemos constatar que muchos no tenían noción de la diversidad y diferencias de vegetación en los ecosistemas amazónicos. Dificultad en entender conceptos como meseta, campos y dinámica de los ríos en la formación del paisaje de los ecosistemas amazónicos.

En la cuestión II, las respuestas no concuerdan con la clase 03. En las cuestiones III y IV, en esa clase que se trataba de estudiar los temas investigados, debatirlos y construir situaciones-problemas que posibiliten respuestas que nos direccionen a construir conceptos relacionados a los temas investigados. Los alumnos trajeron sus investigaciones en papel impreso, libro didáctico o el uso de celular y tablet para estudios. Al principio, la dinámica de división de los textos se concentraba en el líder del grupo o denominado como "el más estudioso", otros grupos dividían las páginas con los otros integrantes del grupo. Siempre que se sentía ociosidad por parte de un grupo o miembros del grupo, el profesor intervino.

En la pregunta $V$, las clases presentaban muchas dudas sobre los significados de enunciados como "epífitas" "campinas" "evapotranspiración" "várzea" "sabanas".

En la cuestión VI, las cuestiones comenzadas en la clase 02 se retomaron porque en la clase 03 era el momento para crear situaciones-problemas.

El profesor estimulaba a los alumnos a responder ¿por qué los bosques tropicales en el mundo se concentraban en una misma posición geográfica? ¿Cuál era la relación de estos ecosistemas con el clima?

Estas situaciones-problemas fueron madurando a medida que la clase 04 de presentación de los trabajos se acercaba.

En la cuestión VII, no coincide la clase 03 porque no hubo presentación de los alumnos.

\subsubsection{Observación de las competencias - Enseñanza 03}

Tabla 13 - Observación de las competencias de los alumnos en la clase 03 de la fase de implementación de la metodología del ABRP

\begin{tabular}{|c|c|c|c|c|c|c|c|}
\hline Requisitos & (A) & $\mathbf{( B )}$ & $\mathbf{( C )}$ & $\mathbf{( D )}$ & $\mathbf{( G )}$ & $\mathbf{( H )}$ & $\mathbf{( I )}$ \\
\hline T1 & $\mathrm{S}$ & $\mathrm{S}$ & $\mathrm{S}$ & $\mathrm{N}$ & $\mathrm{N}$ & $\mathrm{S}$ & $\mathrm{S}$ \\
\hline T2 & $\mathrm{S}$ & $\mathrm{S}$ & $\mathrm{N}$ & $\mathrm{N}$ & $\mathrm{N}$ & $\mathrm{S}$ & $\mathrm{N}$ \\
\hline T3 & $\mathrm{S}$ & $\mathrm{S}$ & $\mathrm{N}$ & $\mathrm{N}$ & $\mathrm{N}$ & $\mathrm{S}$ & $\mathrm{N}$ \\
\hline T4 & $\mathrm{S}$ & $\mathrm{S}$ & $\mathrm{N}$ & $\mathrm{N}$ & $\mathrm{N}$ & $\mathrm{S}$ & $\mathrm{N}$ \\
\hline T5 & $\mathrm{S}$ & $\mathrm{S}$ & $\mathrm{N}$ & $\mathrm{N}$ & $\mathrm{N}$ & $\mathrm{S}$ & $\mathrm{N}$ \\
\hline
\end{tabular}

Los requisitos (E), (F), (J) y (K) no corresponden a la clase 03 .

\subsubsection{Aula 04 y 05}

Figura 4. Aula 04 - Presentación de las situaciones-problemas por los alumnos en la fase de implementación de la metodología del ABRP

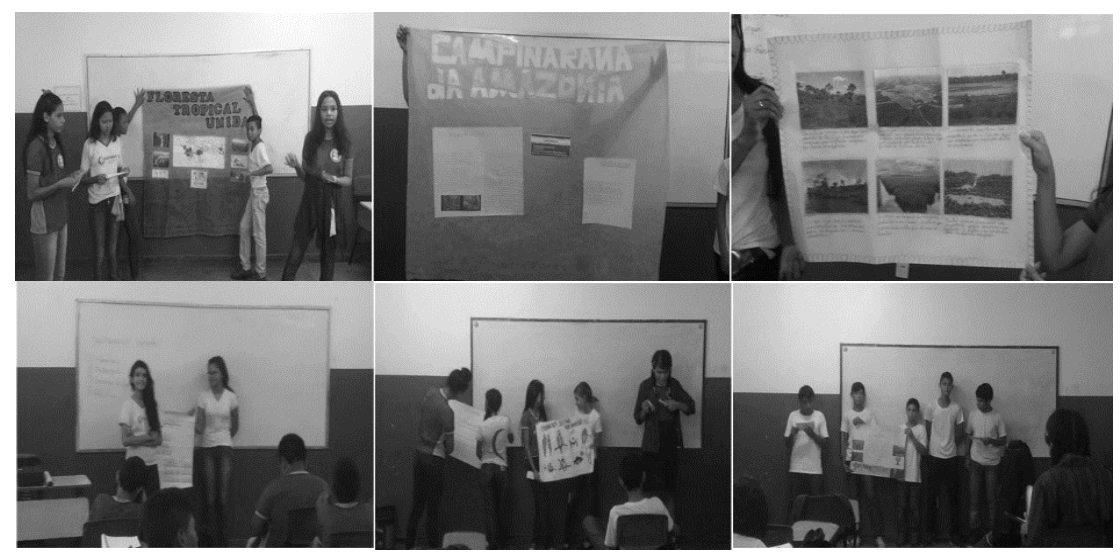

Fonte: Elaborado pelo autor 
En ese proceso de evaluación, cada grupo presentó las soluciones de las situaciones-problemas para los demás grupos, mientras un grupo presentaba, los otros eran orientados para hacer sus anotaciones que fueron respondidas al final de cada presentación. Los grupos adornaron sus presentaciones con carteles o paneles de acuerdo con su tema de estudio.

\subsubsection{Diario del investigador (DI) - clase 04 y clase 05}

El diario del investigador (DI) reveló los siguientes resultados: en la primera parte, cuestión I, de una manera general, los alumnos tuvieron dificultades porque se trataba de un tema con nomenclatura que no era habitual entre los alumnos. Los términos conceptuales en muchos casos eran nominados de una manera que se conocen en nuestra región. Por ejemplo, llamaban "tierras inundadas" a las várzeas.

En la cuestión II, esta cuestión no corresponde a las clases 04 y 05. En las cuestiones III y IV, los líderes y grupos tuvieron autonomía para definir ¿qué criterio adoptarían para elegir al alumno que presentaría el trabajo?

Algunos grupos presentaron todos los componentes de manera limitada. Otros grupos, optaron por elegir un alumno para realizar la presentación, siendo aquel que tuviera una posición más a gusto con el público.

Los problemas de grupo, ocurrieron a medida que los alumnos que se comprometieron a presentar el trabajo, en el momento de la presentación, no se sintieron seguros de realizar la presentación.

En la pregunta V, las dudas que surgieron en el transcurso de las presentaciones, desafortunadamente, algunas ya fueron planteadas en las clases anteriores, como pronunciación de algunos términos o también, comprender la relación posición geográfica y acción climática en la formación de los ecosistemas amazónicos.

En la cuestión VI, las dudas eran acerca de la nomenclatura y pronunciación de términos de los temas investigados.

En la cuestión VII, la mayoría de los grupos presentaron el nerviosismo natural conforme a cada etapa y al momento de presentación al público.

\subsubsection{Observación de las competencias - Enseñanza 04 y 05}

Tabla 14 - Observación de las competencias de los alumnos en la clase 04 de la fase de implementación de la metodología del ABRP

\begin{tabular}{|c|c|c|}
\hline Requisitos & (J) & (K) \\
\hline T1 & S & S \\
\hline T2 & S & N \\
\hline T3 & N & N \\
\hline T4 & N & N \\
\hline T5 & N & N \\
\hline
\end{tabular}

Los requisitos (A), (B), (C), (D), (E), (F), (G), (H), (I), no corresponden a la clase 04 e 05.

5.2.4.3. Re-aplicación del cuestionario sobre conceptos de ecosistemas amazónicos - clase 05

Se revisaron los cuestionarios propuestos después de la implementación de la metodología del ABRP con las mismas cuestiones:

En la cuestión 1, cuando hablamos sobre el ecosistema ¿Qué es lo que usted se imagina?

Los alumnos respondieron de una manera sencilla de acuerdo con su vocabulario, pero no fue posible percibir una forma genérica la respuesta, podemos destacar las diferencias de factores bióticos y abióticos citados. Destacamos algunas respuestas en el tabla 15: 
Tabla 15 - Respuestas de la cuestión 1 en la re-aplicación del cuestionario de las concepciones previas a los alumnos de la Escuela Municipal Jarlece da Conceição Zaranza, Manaos, AM

\begin{tabular}{|l|l|}
\hline ALUNOS & CONCEPÇÕES PRÉVIAS \\
\hline A1 & "Tenemos las várzeas y las sabanas" \\
\hline A5 & "Campos llenos de plantas y animales, ríos que inundan las tierras" \\
\hline A7 & "Ríos, plantas, animales que viven en el lugar con mucha lluvia y calor" \\
\hline
\end{tabular}

En la pregunta 2, los habitantes de Manaos, ¿Qué pensamos sobre la Amazonía?

Se percibió que las respuestas simples del cuestionario antes de la aplicación del ABRP se repitieron, pero en compensación parecen respuestas más objetivas considerando que la Amazonia no es sólo un bosque lleno de plantas y animales, como vemos en las concepciones previas del tabla 16:

Tabla 16 - Respuestas de la cuestión 2 en la re-aplicación del cuestionario de las concepciones previas a los alumnos de la Escuela Municipal Jarlece da Conceição Zaranza, Manaos, AM

\begin{tabular}{|l|l|}
\hline ALUNOS & CONCEPÇÕES PRÉVIAS \\
\hline A1 & "Es un lugar con varios árboles y animales" \\
\hline A5 & "Es donde vivimos, diferentes lugares que llueve mucho, calor, animales y árboles" \\
\hline A7 & $\begin{array}{c}\text { "La Amazonia no es igual, tiene lugares que tienen casas y edificios, otros que sólo } \\
\text { tienen árboles y otros que son inundados por los ríos y las lluvias" }\end{array}$ \\
\hline
\end{tabular}

En la pregunta 3, ¿cuáles son los ambientes de la Amazonía que usted conoce?

Adoptamos presentar los resultados de manera estadística por la repetición de respuestas. En la tabla 17:

Tabla 17 - Respuestas de la cuestión 3 en la re-aplicación del cuestionario de las concepciones previas a los alumnos de la Escuela Municipal Jarlece da Conceição Zaranza, Manaos, AM

\begin{tabular}{|c|c|}
\hline Respuesta & Porcentaje \\
\hline Conozco & $100 \%$ \\
\hline No conozco & $0 \%$ \\
\hline
\end{tabular}

En la tabla 12, muestra que todos los alumnos entrevistados citaron por lo menos uno de los ecosistemas amazónicos que conocen en algún tipo de ambiente en la Amazonia. Siendo $n$ poblacional igual a 100 alumnos entrevistados en esta segunda fase del trabajo.

A continuación, en el tabla 18, qué ambientes conocen los alumnos:

Tabla 18 - Respuestas de la cuestión 3 en la re-aplicación del cuestionario de las concepciones previas a los alumnos de la Escuela Municipal Jarlece da Conceição Zaranza, Manaos, AM

\begin{tabular}{|l|l|}
\hline ALUNOS & CONCEPÇÕES PRÉVIAS \\
\hline A1 & "Várzea alta, várzea baja" \\
\hline A5 & "Campinarana forestal" \\
\hline A7 & "Bosque tropical húmedo y sabana amazónica" \\
\hline
\end{tabular}

En la pregunta 4, ¿has escuchado hablar de un bosque húmedo? Si es así, ¿Cuál es la apariencia de este ecosistema? 
En la tabla 19, los alumnos entrevistados revelan que aprendieron a conocer el bosque tropical húmedo.

Tabla 19 - Respuestas de la cuestión 4 en la re-aplicación del cuestionario de las concepciones previas a los alumnos de la Escuela Municipal Jarlece da Conceição Zaranza, Manaos, AM

\begin{tabular}{|l|c|}
\hline Respuesta & Porcentaje \\
\hline Si & $100 \%$ \\
\hline No & $0 \%$ \\
\hline
\end{tabular}

En el tabla 20, muestra sólo las respuestas de aquellos alumnos que ya escucharon hablar del bosque húmedo destacando ¿Cuál es su apariencia?

Tabla 20 - Respuestas de la cuestión 4 en la re-aplicación del cuestionario de las concepciones previas a los alumnos de la Escuela Municipal Jarlece da Conceição Zaranza, Manaos, AM

\begin{tabular}{|l|l|}
\hline ALUNOS & CONCEPÇÕES PRÉVIAS \\
\hline A1 & "Es la mayor selva tropical del mundo, donde se encuentran animales, lagos y peces" \\
\hline A5 & "Es una de las mayores biodiversidades del mundo" \\
\hline A7 & "Está bajo protección del gobierno, donde existen las reservas naturales" \\
\hline & "Llueve todo el año y los árboles se inclinan para el uso de la madera" \\
\hline
\end{tabular}

En la pregunta 5, ¿has escuchado hablar de várzea? Si es así, ¿Cuál es la apariencia de este ecosistema?

En la tabla 21, muestra que todos los alumnos entrevistados en el total de 100, aprendieron las características de una várzea.

Tabla 21 - Respuestas de la cuestión 5 en la re-aplicación del cuestionario de las concepciones previas a los alumnos de la Escuela Municipal Jarlece da Conceição Zaranza, Manaos, AM

\begin{tabular}{|l|c|}
\hline Respuesta & Porcentaje \\
\hline Si & $100 \%$ \\
\hline No & $0 \%$ \\
\hline
\end{tabular}

En la pregunta 6, ¿has escuchado hablar de campinaranas? Si es así, ¿Cuál es la apariencia de este ecosistema?

En la tabla 22, muestra que el 100\% de los alumnos entrevistados, pudieron conocer el ecosistema amazónico llamado campinarana.

Tabla 22 - Respuestas de la cuestión 6 en la re-aplicación del cuestionario de las concepciones previas a los alumnos de la Escuela Municipal Jarlece da Conceição Zaranza, Manaos, AM

\begin{tabular}{|c|c|}
\hline Respuesta & Porcentaje \\
\hline Si & $100 \%$ \\
\hline No & $0 \%$ \\
\hline
\end{tabular}

En la pregunta 7, ¿has escuchado hablar de sabanas? Si es así, ¿Cuál es su apariencia? 
En la tabla 23, muestra que el 100\% de los alumnos entrevistados, conocen o han escuchado hablar en sabanas, comprendiendo que existe no sólo la sabana africana.

Tabla 23 - Respuestas de la pregunta 7 en la re-aplicación del cuestionario de las concepciones previas a los alumnos de la Escuela Municipal Jarlece da Conceição Zaranza, Manaos, AM

\begin{tabular}{|c|c|}
\hline Respuesta & Porcentaje \\
\hline Si & $100 \%$ \\
\hline No & $0 \%$ \\
\hline
\end{tabular}

El encuentro 06, la visita a la Reserva Forestal Adolpho Ducke, aún no fue posible realizarla por tratarse de un espacio privado, como también no conseguimos los recursos necesarios para el alquiler de autobuses e ingresos a la reserva, además de la logística de llevar 100 alumnos para ese lugar.

El encuentro 07, quedó comprometido debido al retraso del encuentro 06, esperamos en el futuro próximo cumplir con esas formalidades que no incidieron sobre los datos recolectados.

\section{Conclusión}

Este es el momento de comprender si los resultados obtenidos en la investigación explican en alguna dimensión los objetivos definidos y responden a las cuestiones orientadoras del trabajo. Es posible poner a disposición una alternativa metodológica para el aprendizaje de conceptos relacionados con los ecosistemas amazónicos. Los instrumentos de recolección de datos revelaron que después de la implementación del aprendizaje basado en la resolución de problemas, los alumnos lograron revertir datos de las concepciones previas.

Las respuestas en las concepciones previas eran netamente lógicas y repetidas a través de los enunciados adquiridos en los medios de comunicación en masa, los cuales transmitían una visión de Amazonia mítica, sobreestimada y homogénea. Después de la implementación, las respuestas revelaron una visión de la Amazonia más conceptual con amplia biodiversidad. Las respuestas después de la implementación, demuestran que los alumnos comprenden que la Amazonia no son solamente ríos, animales y plantas. Actualmente presenta problemas de asentamientos ilegales, y su riqueza de recursos naturales se explota con fines de lucro comercial.

Un cambio significativo y perceptible se dio en el porcentaje de alumnos que conocían los ambientes en la Amazonia. Todos los alumnos después de la implementación del ABRP conocían al menos uno de los tipos de ecosistemas. Mientras que antes de la implementación del ABRP, sólo el $65 \%$ admitió conocer al menos un ambiente.

En cuanto a los ambientes que eran conocidos en la Amazonia antes de la implementación del ABRP, las respuestas eran muy genéricas y se resumía la apariencia homogénea de la Amazonia. Como si se limitara a un bosque tropical húmedo. Después de la implementación del ABRP, los alumnos lograron citar ecosistemas amazónicos diferentes en sus paisajes y formación geológica.

Cuando se trata de los ecosistemas amazónicos Várzea, Campinarana y Sabana, los alumnos desconocían totalmente esos ambientes, sin siquiera, nunca haber escuchado hablar de ninguno de ellos. Causa extrañeza, ¿cómo es que la juventud que vive en la Amazonia, no conocen su propia casa?

Después de la implementación del ABRP, los alumnos no sólo conocían en su mayoría los ambientes, sino que también conseguían diferenciar y clasificar esos ecosistemas, adicionalmente, fueron citadas divisiones como "várzea alta y baja" y "campinarana forestal". Comenzaron a comprender cómo la dinámica de los ríos está relacionada con la variación climática. O incluso, con 
la posición geográfica y los movimientos de rotación y traslación de la tierra. Comenzaron a comprender lo difícil que es diferenciar esos ambientes inmersos en el bosque, pues podemos adentrarnos en un bosque tropical húmedo, caminar un poco y encontrarnos con un campo abierto o un terreno inundado.

En cuanto a los instrumentos de recolección de datos, de acuerdo con cada clase de la implementación del ABRP, ellos nos revelaron una dificultad natural de interactuar con otros miembros del grupo, distribución de tareas y con la madurez para liderar el grupo. Los alumnos tuvieron dificultades para participar en una actividad donde tenían autonomía para realizar las acciones, a diferencia de las prácticas pedagógicas centradas en el profesor.

Con el uso del instrumento de recolección de datos en la fase de concepciones previas, se puede cumplir en alguna medida el conocimiento del contenido del discurso de los alumnos sobre los ecosistemas amazónicos. Así como, asignar el uso del ABRP para verificar un cambio en el discurso antes y después de la implementación del ABRP. Es importante resaltar, que prácticas pedagógicas influencian en el aprendizaje de los alumnos en distintos períodos de tiempo, tal vez, en el futuro será necesario un período mayor de práctica de campo para asegurar la continuidad de esos conceptos.

Las dificultades eran dominio técnico del ABRP, aunque no se tuvo la oportunidad de ir hasta Portugal o incluso interactuar con otro profesional que hubiese utilizado el ABRP en la investigación pedagógica. La literatura en torno al tiempo no es muy amplia en relación a los detalles en la construcción de las situaciones-problema, conducción pedagógica del proceso o análisis de los datos.

Otra dificultad encontrada está en el uso del espacio formal para la realización de la práctica de campo, el acceso a la escuela, la disponibilidad de alumnos, la cesión de horario alternativo que no altere la dinámica de la escuela. Así como la motivación a los alumnos en el sentido de convencer sobre la importancia de la investigación.

Otra dificultad encontrada que es importante mencionar, se dio por las adaptaciones de los instrumentos de recolección de datos, pues se trataba de materiales producidos en una realidad distinta de las encontradas en las escuelas del municipio de Manaos. Para registrar las competencias individuales de los alumnos eran necesarios más profesionales o auxiliares, pues estamos hablando de un universo de 100 alumnos.

Este trabajo no tuvo la intención de agotar el tema sobre el uso del ABRP, ni siquiera los resultados no se aplican a una realidad diferente de la encontrada en el mundo de la investigación. También, las conclusiones no se aplican a generalizar sobre ¿̇cómo los alumnos aprenden conceptos relacionados a los ecosistemas amazónicos? o se aplican sobre ¿cómo los jóvenes que viven en la Amazonia conocen su contexto forestal? Al final, 100 alumnos no representan un universo de más de 1 millón de alumnos matriculados como demuestra el último censo escolar de 2017.

Es necesaria una continuidad del trabajo en los años que vienen, con apoyo financiero para contratar profesionales o auxiliares para atender un universo mayor. El ideal sería una muestra del $5 \%$ de los alumnos matriculados en todo el Amazonas. En ambientes distintos, contrastar el municipio de Manaos con otros municipios más distantes del perímetro urbano. Sería interesante, una etapa en Portugal por el dominio técnico en la aplicación del ABRP.

Se espera que esta iniciativa sea el primer paso en instrumentalizar a nuestros jóvenes en la construcción de saberes sobre los ecosistemas amazónicos, con el fin de contribuir a la formación de una generación consciente de su responsabilidad con el medio ambiente y con la preservación y conservación de nuestra Amazonia.

\section{Agradecimentos}

A las instituciones Universidad del Estado de Amazonas (UEA), Universidad Federal del Amazonas (UFAM) y la Fundación de Amparo a la Investigación del Estado de Amazonas (FAPEAM), Red Bionorte y Secretaría Municipal de Educación de Manaus, AM. 


\section{Referências}

AUBRÉVILLE, A. Essai de classification et de nomenclature des formations forestières africaines avec extension du système propose à toutes les formations du monde tropical. In: CSA SPECIALIST MEETING ON PHYTO-GEOGRAPHY, Yangambi, Congo, 1956:247-288.

AZEVEDO, A. Regiões climato-botânicas do Brasil. Boletim Paulista de Geografia, São Paulo: Associação dos Geógrafos Brasileiros - AGB-SP. 1950;6(1):32-43.

BARROWS, H., \& TAMBLYN, R. Problem-based learning: An approach to medical education. Nova Iorque: Springer. 1980.

BEARD, J. S. The classification of tropical American vegetation-types. Ecology. 1955;36:89-100.

BELT, S. T.; EVANS, E. H; MCREDDY, T.; OVERTON, T.L., \& SUMMERFIELD, S. A. Problem-based learning approach to analytical and applied chemistry. University Chemistry Education. 20026:65-72.

BERBEL, Neusi (org.). Metodologia da Problematização: Fundamentos e aplicações. Ed. UEL. 1999.

BOUJAOUDE, S. The relationship between students' learning strategies and the change in their misunderstandings during a high school chemistry course. Journal of Research in Science Teaching, 29(7), 687-699. 1992.

BROWN, Stuart. 100 Filósofos do Século XX. Instituto Piaget. 2002.

BURTT-DAVY, J. The classification of tropical woody vegetation types. Oxford: University of Oxford, Imperial Forestry Institute. 1938;13:85.

CAMPOS, G. de. Mapa florestal do Brasil. Rio de Janeiro: Ministério da Agricultura, Indústria e Comércio, Typ. do Serviço de Informações. 1926:147.

CARVALHO, C. ; DOURADO, L. A formulação de questões a partir de cenários problemáticos: um estudo com alunos de Ciências Naturais do $3^{\circ}$ Ciclo do Ensino Básico português. In Atas do X Congresso Internacional Galego-Português de Psicopedagogia. Braga: Universidade do Minho. 2009:2615-2628.

CHANDRASEGARA, A., TREAGUST, D. \& MOCERINO, M. An evaluation of Teaching Intervention to Promote Students' Ability to Use Multiple Levels of Representation when describing and explaining chemical reactions. Research in Science Education. 2008;38:237-248.

CHANG, SW. A review of literature on new pathway medical education. News New Curri CGU.2002;1:3249.

COLL, César; SOLE, Isabel. Os professores e a concepção construtivista. In: COLL, César et al. O Construtivismo na sala de aula. 4 ed. São Paulo: Ática. 1998:9-28.

COLE, M. M. The savannas: biogeography and geobotany. London: Academic Press, 1986. 438 p.

COLE, M.M. Vegetation nomenclature and classification with particular reference to the savannas. South African Geographical Journal, Bloemfontein [África do Sul]: Society of South African Geographers - SSAG, v. 55, p. 3-14, 1963.

DAHLGREN, M. \& ÖBERG, G. Questioning to learn and learning to question: structure and function of problem based learning scenarios in environmental science education. Higher Education. 2001;41:263-282. 
DANSEREAU, P. Introdução à Biogeografia. Revista Brasileira de Geografia. 1968;1: 1-85.

DEWEY, J. Experience and Education. Toronto: Collier-MacMillan Canada Ltd. 1938.

DI GREGORIO, A. Land cover classification system (LCCS): classification concepts and user manual. Version 2.Rome: Food and Agriculture Organization of the United Nations - FAO. 2005;190.

DODS, R. A problem-based learning design for teaching biochemistry. Journal of Chemical Education. 1996;73:225-228.

EITEN, G. Classificação da vegetação do Brasil.Brasília, DF: Conselho Nacional de Desenvolvimento Científico e Tecnológico - CNPq, 1983. 305 p.

ELLENBERG, H.; MUELLER-DOMBOIS, D. A key to raunkiaer plant life-forms with revised subdivisions. Berichte des Geobotanischen Institutes der Eidg. Techn. Hochshule Stiftung Rübel, Zurich: ETH. 1967;37:56-73.

FERNANDES, A. G. Fitogeografia brasileira. Fortaleza: Multigraf, 1998:339.

Fundação Instituto Brasileiro de Geografia Estatística-IBGE. Mapa de vegetação do Brasil. Mapa 1:5,000,000. Rio de Janeiro, Brasil. 1993.

GÜRSES, A., ACIKYILDIZ, M., DOAR, Ç. \& SÖZBILIR, M. An investigation of effectiveness of problembased learning at physical chemistry laboratory. Research in Science and Technological Education.2007;78:1126-1130.

HUFFMAN, D., LAWERNZ, F. \& MINGER, M. Within-class analysis of ninth-grade science students' perceptions of the learning environment. Journal of Research in Science Teaching. 1997;34(8);791-804.

KUHLMANN, E. Vegetação. In: GEOGRAFIA do Brasil. Rio de Janeiro: IBGE, 1977;1:59-94.

LAMBROS, A. Problem-Based Learning in K-8 classrooms. Thousand Oaks: Corwin Press. 2002.

LEITE, L. ; PALMA, C. Formulação de questões, educação em ciências e aprendizagem baseada na resolução de problemas: Um estudo com alunos portugueses do $8^{o}$ ano de escolaridade. In Atas do Congresso PBL 2006 ABP (CD-Rom). Lima (Peru): Universidade Pontifícia Católica del Peru. 2006.

MEADE,R.H. Suspended sediments of the modern Amazon and Orinoco Rivers. Quaternary Research. 1994;21:29-39.

MORRONE, J. J. Biogeografía de América Latina y el Caribe.Zaragoza [Espanha]: Sociedad Entomológica Aragonesa - SEA, 2001:148.

OSTERMAN, K. \& KOTTKAMP, R. Reflective Practice for Educators. Newbury Park, California: Sage. 1993.

PRANCE, G.T. Notes on the vegetation of Amazonia III. The terminology of Amazonian forest types subject to inundation. Brittonia, 1979, 3(1):26-38.

PROJETO RADAM BRASIL - Sítio Eletrônico dos Antigos Funcionários do Projeto Radam Brasil. Disponível em:<http://www.projeto.radam.nom.br/index.html>. Acesso em: 08 out. 2010.

RIZZINI, C. T. Árvores e madeiras úteis do Brasil: manual de dendrologia brasileira. São Paulo: E. Blücher. 1979:294. 
SAMPAIO, A. J. Fitogeografia. Revista Brasileira de Geografia, Rio de Janeiro: IBGE, ano 2, 1943;1:59-78.

SCHIMPER. Plant-geography upon a physiological basis. Oxford: Clarendon Press. 1903:839.

TANSLEY, A. G.; CHIPP, T. F. (Eds.). Aims and methods in the study of vegetation. London: Crown. 1926:349-361.

TARHAN, L., AYAR-KAYALI, H., UREK, R. O. \& AZAR, B. Problem-based Learning in 9th Grade Chemistry Class: Intermolecular Forces. Research in Science Education. 2008; 38:285-300.

TAYLOR, J. A. \& MCDONALD, C. Writing in groups as a tool for non-routine problem solving in first year university mathematics. International Journal of Mathematics Education in Science. 2007;38 (5): 639655 .

TROCHAIN, J. L. Accord interafricain sur la définition des types de végétation de l'Afrique tropicale, Bidl. I.E.C. 1969;(13-14):55-93.

VASCONCELOS, C. Aprendizagem Baseada na Resolução de Problemas: Um estudo no âmbito da Educação Ambiental. Relatório de Pós-Doutoramento. Braga: Instituto de Educação e Psicologia da Universidade do Minho. 2008.

VASCONCELOS, C. Teaching Environmental Education through PBL: Evaluation of a Teaching Intervention. Program. Research in Science Education. 2012;42(2): 219-232.

WHITE, H. B. A. PBL course that uses research articles as problems. 2001. 